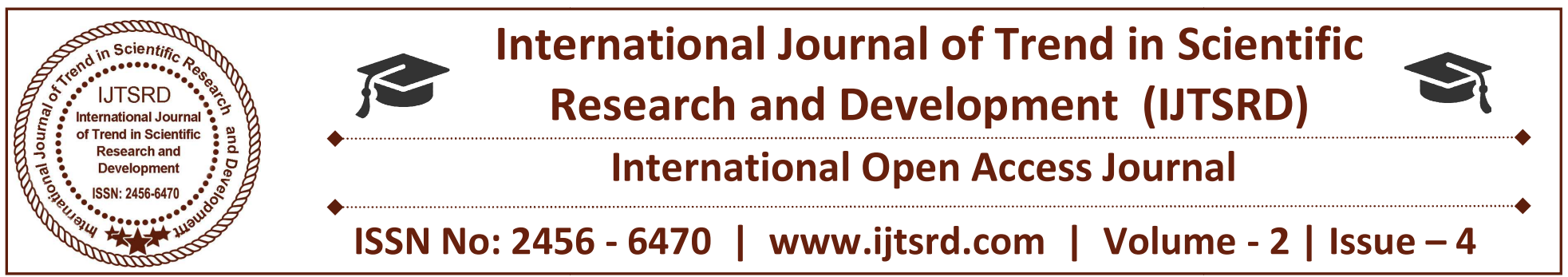

\title{
Comparative Analysis of Dispersion in Decagonal Structure of Photonic Crystal Fiber having Hole within Core
}

\author{
Shivani Gangwal ${ }^{1}$, Vipra Bohara $^{2}$, Laxmi Narayan Balai ${ }^{3}$ \\ ${ }^{1}$ P.G. Scholar, ${ }^{2}$ Assistant Professor, ${ }^{3}$ H.O.D. \\ Department of Electronics \& Communication, Yagyavalkya Institute of Technology, \\ Jaipur, Rajasthan, India
}

\section{ABSTRACT}

This paper comparatively analyzes decagonal 4 cladding ring structure having circular \& square hole within core and structure without hole in core. All simulations have been performed in COMSOL Multiphysics simulation tool. Effective refractive index is calculated for each design by varying pitch keeping cladding air hole diameter constant and by varying air hole diameter keeping pitch constant. Dispersion is calculated using finite element method. Optimized design is obtained by comparing all the designs.

Keywords: Dispersion, Finite element analysis, Photonic crystal fiber, Wavelength

\section{INTRODUCTION}

The idea of photonic crystals propagated in 1987 with effort in the field of the strong localization of light, and for the inhibition of spontaneous emission. It was afterward shown that in periodic arrangements of ideally lossless dielectric material, the transmission of light can be totally suppressed at certain wavelengths, in any case of propagation direction and polarization. Such kinds of periodic arrangements of dielectric materials have been known as photonic crystals. The first fiber with a photonic crystal structure was observed by Philip St. J. Russell in 1995. Photonics is the branch of modern science and technology that link electronics and optics and often deals with the generation, propagation, and detection of light. Thus, photonics can manage the photons.
In the solid core MOFs that we will learn, light direction is due to customized total inner indication among the center and a micro prepared cladding consisting of inclusion in a matrix. The center and matrix material are normally the same, and hence have the same refractive index. In put into practice, the cladding has a fixed width, as it consists of a number of rings of inclusions. Ahead of the micro structured part of the fiber, the matrix extends devoid of any inclusions awaiting the jacket is reached. If we think about the jacket to be far from the cladding and core, and hence overlook its influence, supervision in the core is solely due to a finite number of layers of holes in bulk silica extending to infinity. A priori, the cladding does not "protect" the core from the surrounding matrix substance since the holes do not amalgamate with their neighbours and therefore the matrix is connected between the core and the exterior. Physically, we can visualize the light leaking from the central part to the exterior matrix material through the bridges between holes, and thus expect losses. In the modified total interior reflection model of guidance, in which the micro structured part of the fiber is replaced by standardized material with an efficient refractive index lower than the core, the core is entirely surrounded by the cladding. The peripheral matrix material and the core are then no longer directly connected. On the other hand the thickness of the "effective cladding" is fixed, and hence tunnelling losses are obvious. Despite of the approach one uses to explain guidance in MOFs, as extensive as guidance is due to a fixed number of layers of holes, 
leakage from the core to the external matrix material is obligatory.

Photonic crystals present several stimulating opportunities for increasing new optical apparatus for optical interactions [4-5]. The crystal structures are well-known for their potentials for confine and directing light in very minute structures. Photonic crystals are able to also display strong diffusion properties that may perhaps give rise to large group delay, strong chromatic dispersion, and major polarization-dependent diffusion for pulse proliferation. Photonic crystals offer stimulating potentials for key performing techniques in optical communication systems, such as delay elements, chromatic dispersion compensators, polarization mode dispersion mitigates and wavelength adjoin/fall filters.

Photonic crystal fiber is less costly than copper wires. Since optical fibers are narrower than copper wires, additional fibers can be bundled into any -width cable than copper wires. This allows more receiver lines to go in excess of the same cable. The loss of signal in photonic crystal fiber is less. It is preferably suitable for carrying digital information, which is especially helpful in computer networks. It is free from fire hazard as no electricity can pass through it. It is smaller in diameter.

\section{PCF DESIGNS}

We have designed 3 types of structures of photonic crystal fiber: (i) Decagonal structure (ii) Decagonal structure with circular hole in core and (iii) Decagonal structure with square hole in core. We have designed four rings in cladding area with perfectly matched layer surrounding the cladding. Core is made of silica and air is filled in cladding. Designs are shown in Fig $1,2 \& 3$.

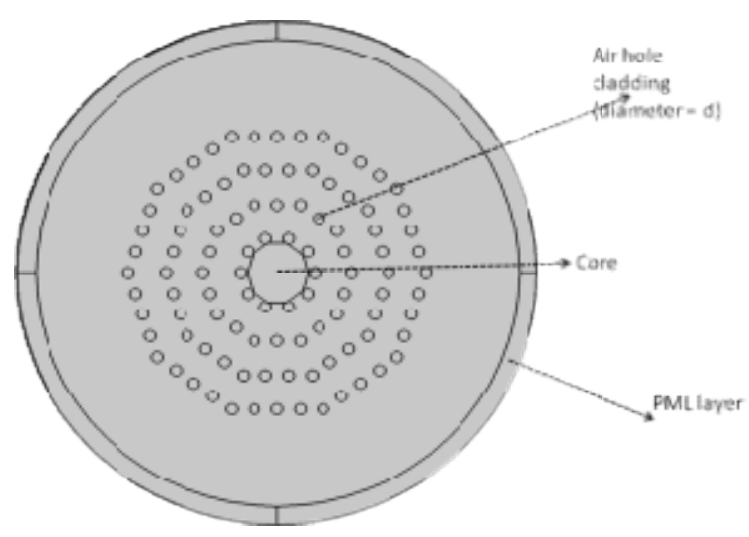

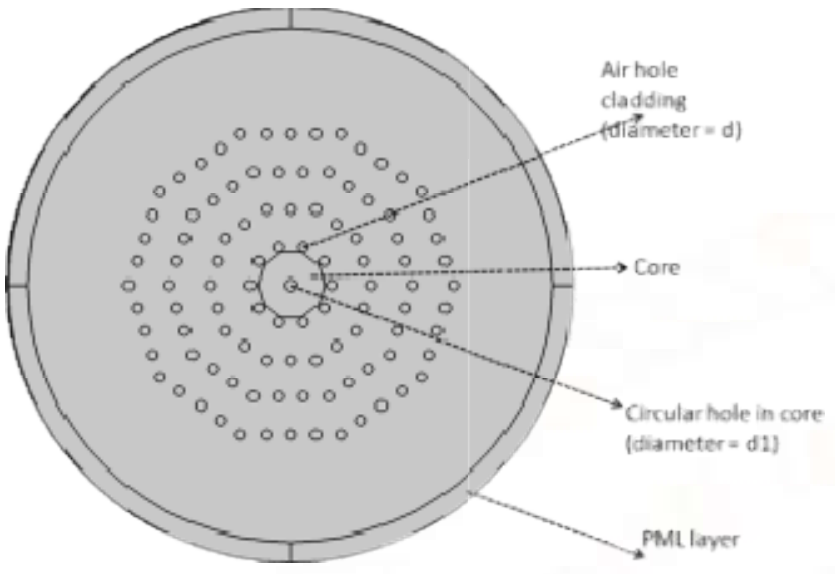

Fig 2 Decagonal 4 ring structure with circular hole in core

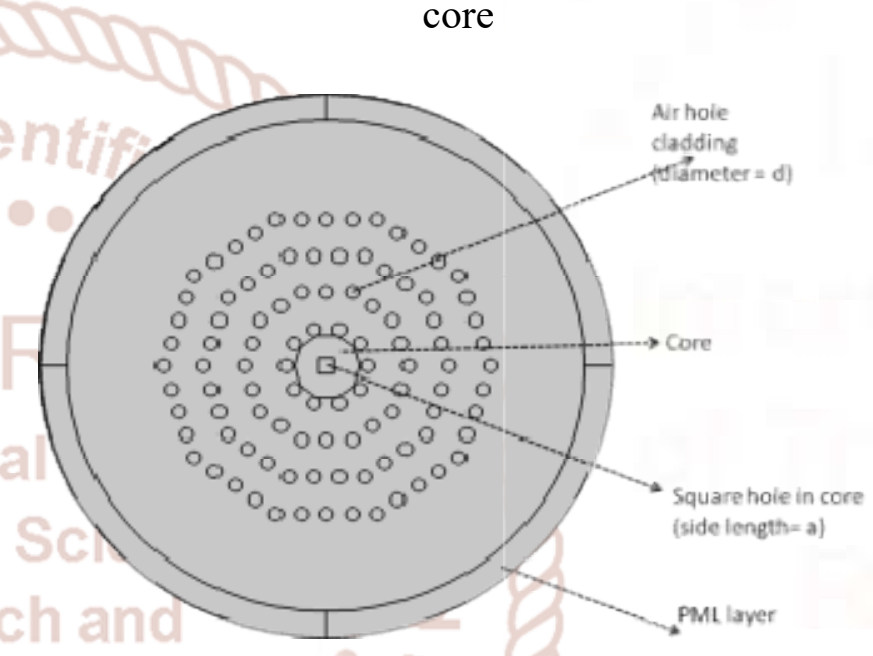

Fig 3 Decagonal 4 ring structure with square hole in core

In above designs diameter of cladding air hole is $\mathrm{d}$ and spacing between two air holes is pitch. Diameter of circular hole is $\mathrm{d} 1$ which is constant and side length of square hole is a, which is also kept constant.

\section{ANALYSIS OF PCF BASED ON EFFECTIVE REFRACTIVE INDEX}

PCF designs are analysed based on effective refractive index by varying pitch keeping air hole diameter $(0.5 \mu \mathrm{m})$ constant and by varying air hole diameter keeping pitch $(2 \mu \mathrm{m})$ constant at different values of wavelength. Diameter of circular hole is 0.6 $\mu \mathrm{m}$ and side length of square hole is $0.6 \mu \mathrm{m}$.

Fig 1 Decagonal 4 ring structure 
International Journal of Trend in Scientific Research and Development (IJTSRD) ISSN: 2456-6470

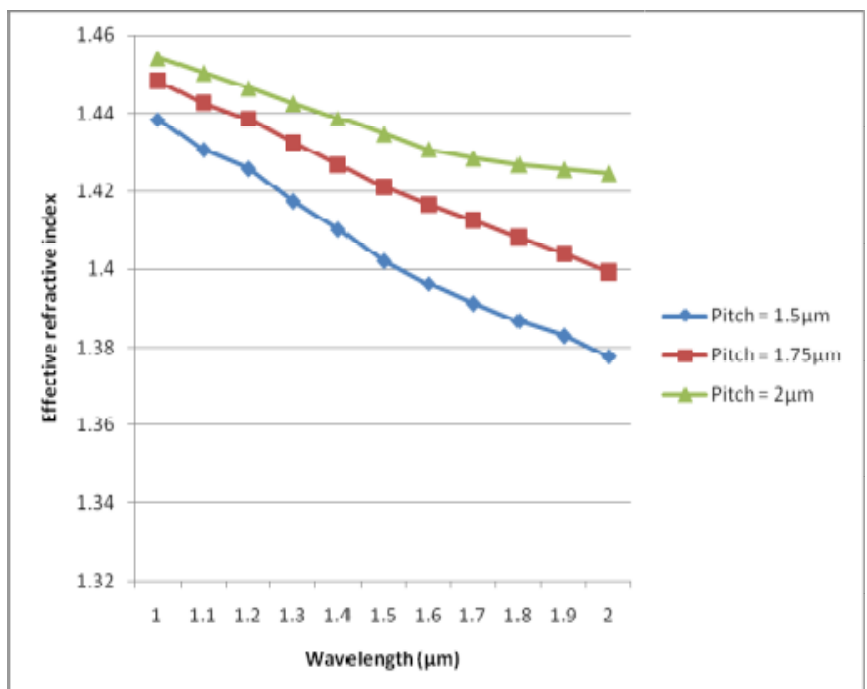

Fig 4 Effective refractive index for different pitch in PCF

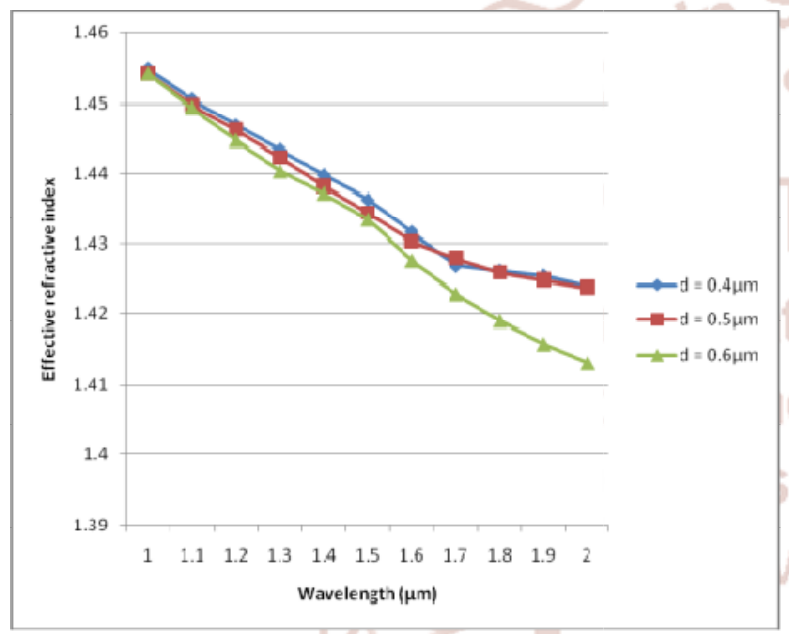

Fig 5 Effective refractive index for different diameter in PCF

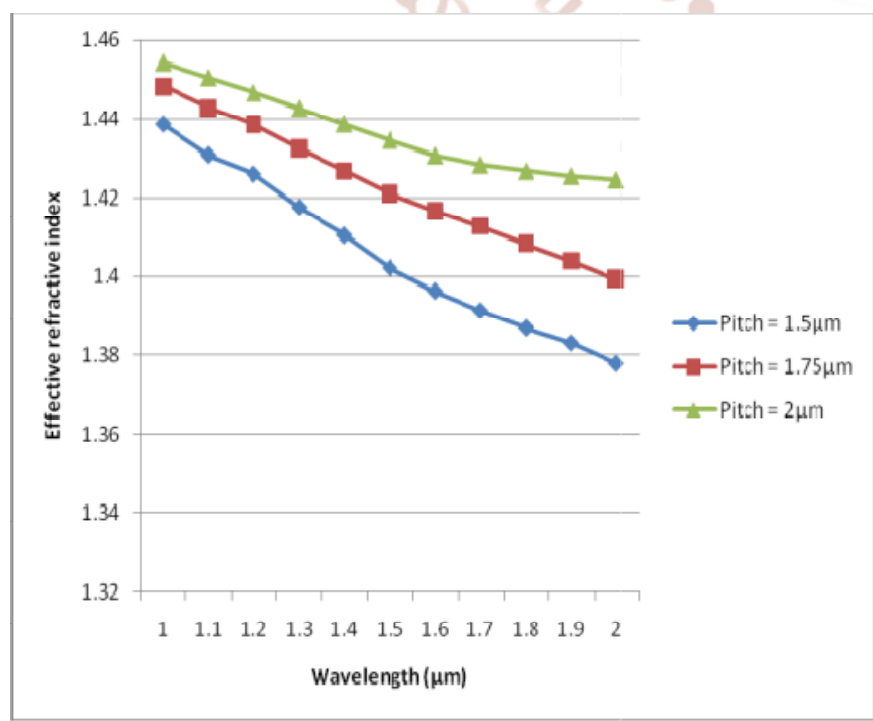

Fig 6 Effective refractive index for different pitch in PCF (circular hole)

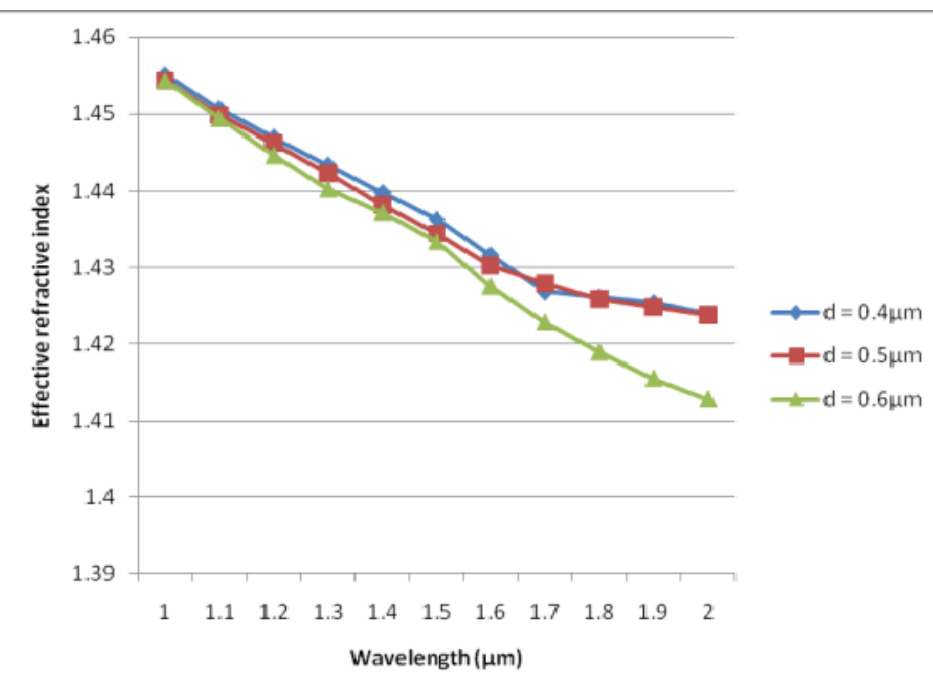

Fig 7 Effective refractive index for different diameter in PCF (circular hole)

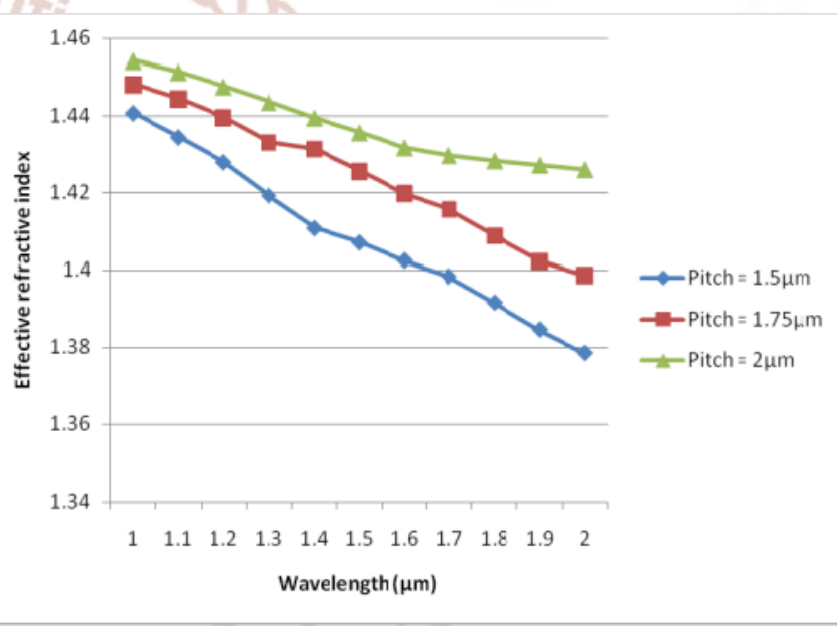

Fig 8 Effective refractive index for different pitch in PCF (square hole)

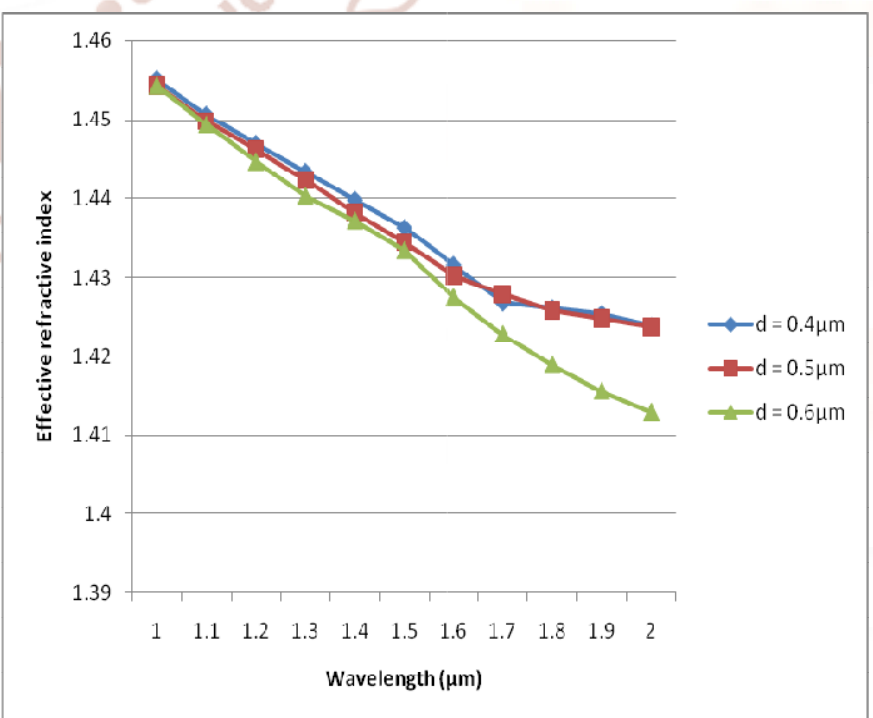

Fig 9 Effective refractive index for different diameter in PCF (square hole) 
International Journal of Trend in Scientific Research and Development (IJTSRD) ISSN: 2456-6470

\section{ANALYSIS OF PCF BASED ON DISPERSION}

PCF designs are analysed based on dispersion by varying pitch keeping air hole diameter $(0.5 \mu \mathrm{m})$ constant and by varying air hole diameter keeping pitch $(2 \mu \mathrm{m})$ constant at different values of wavelength.

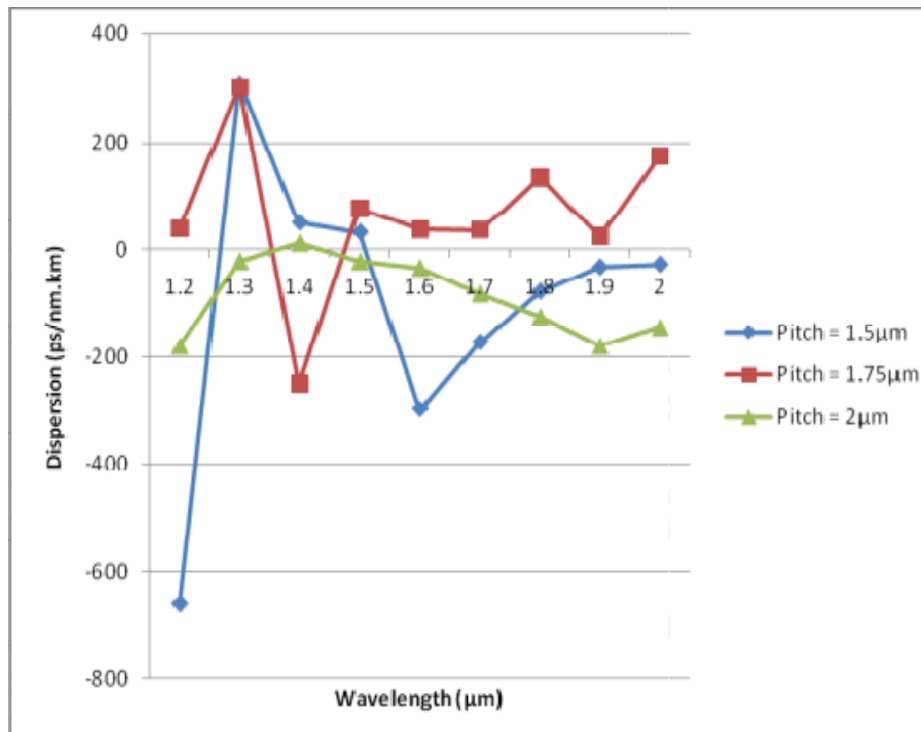

Fig 10 Dispersion for different pitch in $\mathrm{PCF}$

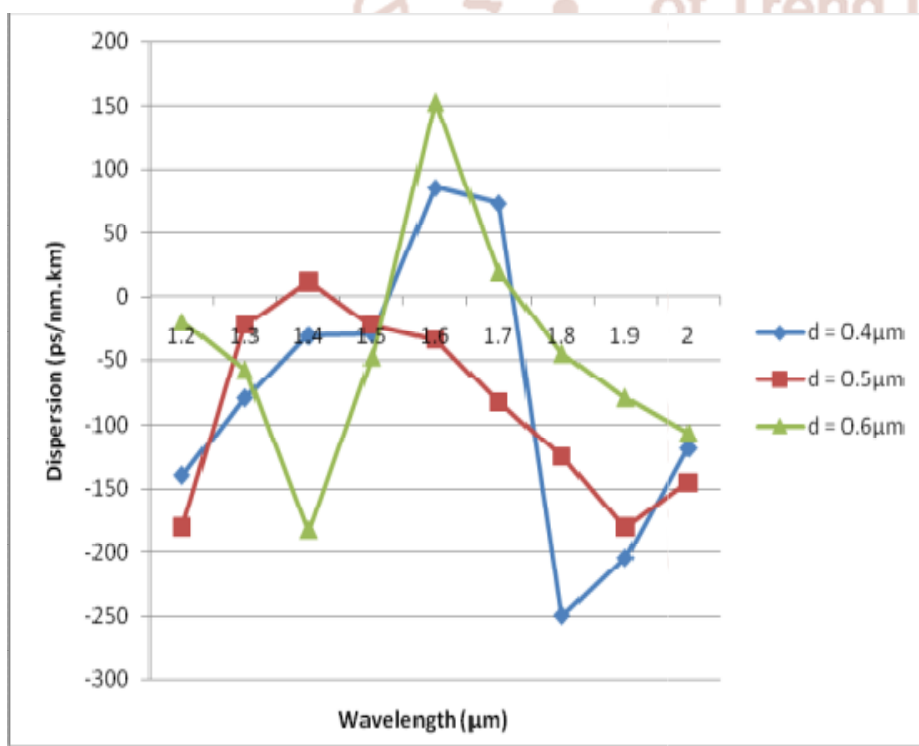

Fig 11 Dispersion for different diameter in PCF

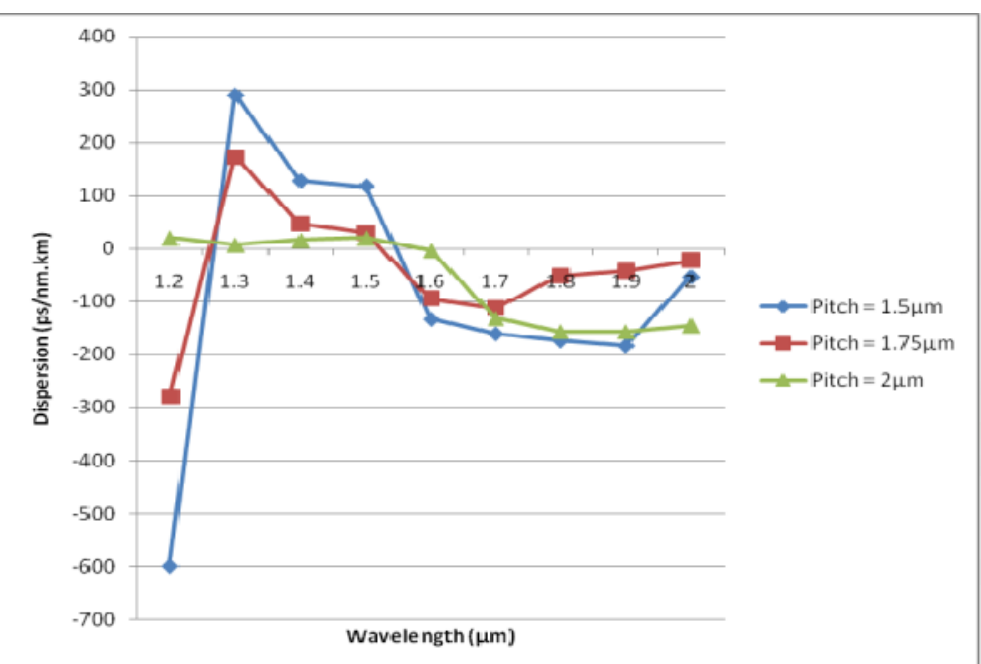

Fig 12 Dispersion for different pitch in PCF (circular hole)

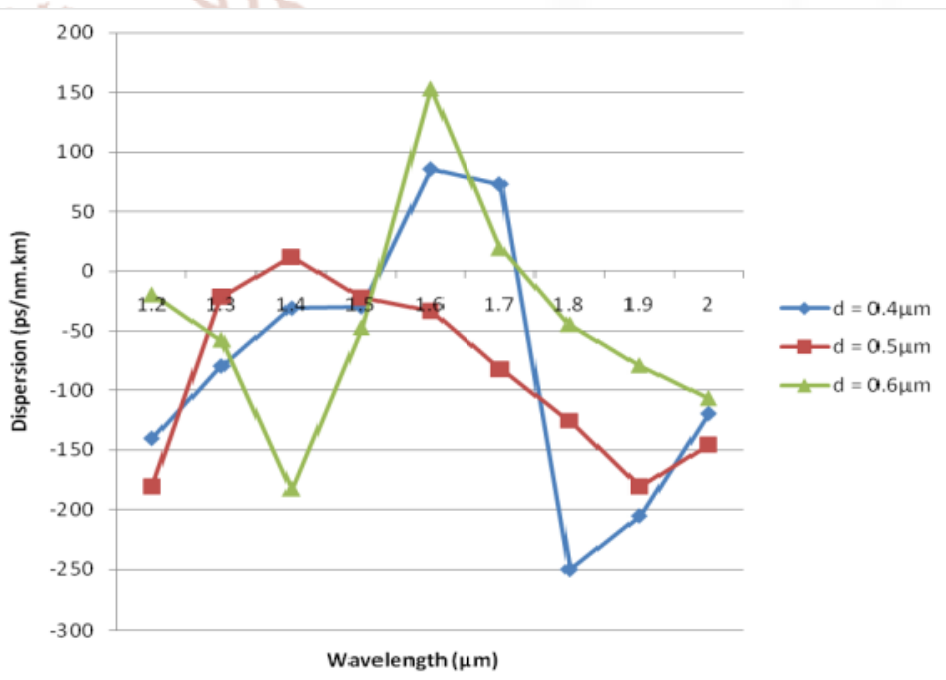

Fig 13 Dispersion for different diameter in PCF

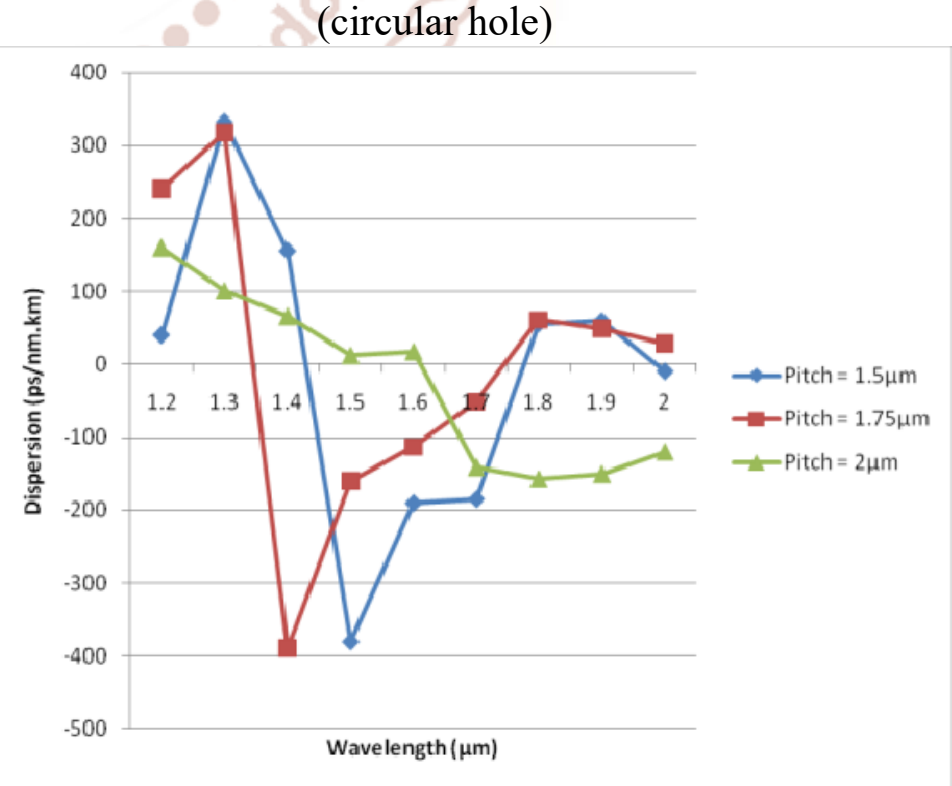

Fig 14 Dispersion for different pitch in PCF (square hole) 


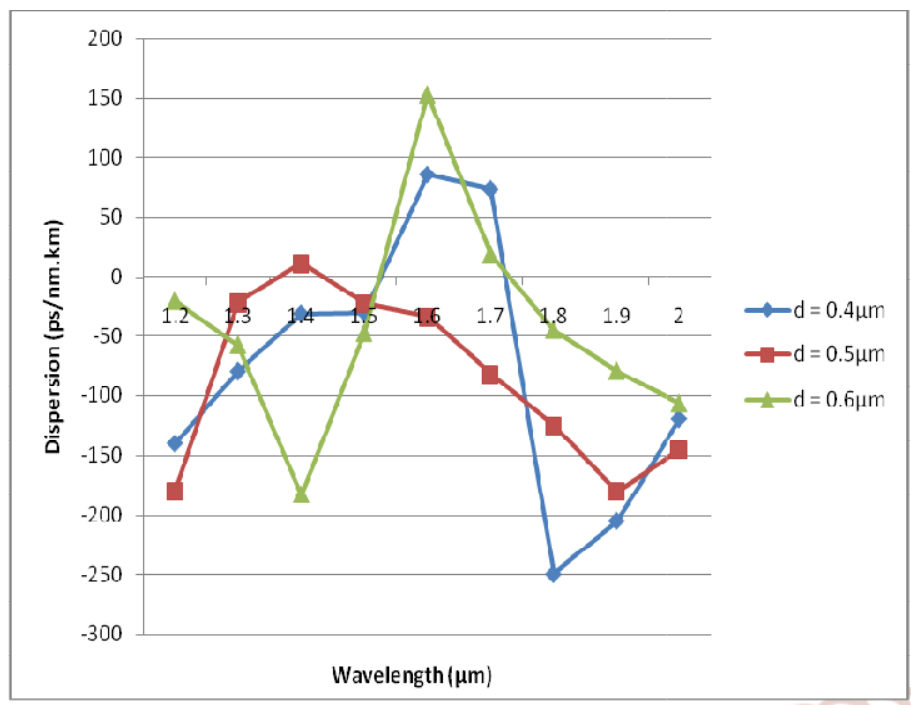

Fig 15 Dispersion for different diameter in PCF (square hole)

\section{CONCLUSION}

According to our observations, optimized values of pitch and air hole diameter are $1.75 \mu \mathrm{m}$ and $0.5 \mu \mathrm{m}$ respectively. At these values PCF with square hole in core produces better values of effective refractive index but in case of dispersion, this structure produces high dispersion at lower wavelengths and low dispersion at higher wavelengths as compared to other designs. In future various other designs of decagonal structure can be compared using COMSOL or other simulation tools. Besides effective refractive index and dispersion, other factors like confinement loss, propagation constant etc can be computed according to different affecting factors.

\section{REFERENCES}

1) Fetah Benabid and Peter J. Roberts, "Hollow Core Photonic Crystals Fibers: Fundamental and Applications", Taylor \& Francis, 2011.

2) Shubi F. KAIJAGE, Yoshinori NAMIHIRA, Nguyen H. HAI, Feroza BEGUM, S. M. Abdur RAZZAK, Tatsuya KINJO, Hiroki HIGA, and Nianyu ZOU, "Multiple Hole-core Hexagonal
Photonic Crystal Fiber with Flattened Dispersion and Polarization Maintaining Properties", Optical Review, 2008.

3) Yuan-Fong Chau, "Design of a high-birefringence photonic crystal fiber using an asymmetric hole in the fiber core", IEEE, 2014.

4) Mukesh Nagar, Yash Walia, Sukoon Mishra, Abhay Dixit, Yuvraj Handa, "Nearly Zero Flattened chromatic dispersion in five layer hole core photonic crystal fibre", International Journal of Innovative Research in Computer and Communication Engineering, 2013.

5) Namassivayane Kejalakshmy, B. M. Azizur Rahman, Arti Agrawal, Huda M. Tanvir, and Kenneth T. V. Grattan "Metal-Coated Hole-Core Photonic Crystal Fiber for $\mathrm{THz}$ Propagation" IEEE, 2009.

6) Md. Sajjad Hossain, Kishan Neupane, Md. Shihab Bin Hafiz, and Satya Prasad Majumder, "Dispersion and Nonlinear Characteristics of a Photonic Crystal Fiber (PCF) with Holeed Core and Various Doping Concentration", International Conference on Electrical and Computer Engineering, 2014.

7) Venimadhav Sharma, Sachin Chauhan and Sunil Sharma, "Proposed Novel Design of Silica PCF to Minimizing the Dispersion Using OptiFDTD", International Conference on Recent Advances and Innovations in Engineering, IEEE, 2016.

8) Animesh Bala Ani, and Mohammad Faisal, "Ultra-flattened Broadband Dispersion Compensating Photonic Crystal Fiber with Ultralow Confinement Loss", International Conference on Electrical and Computer Engineering, IEEE, 2016. 